\title{
Acknowledgement of manuscript reviewers 2016
}

\author{
Panagiotis Behrakis ${ }^{1,2}$
}

\section{CONTRIBUTING REVIEWERS}

The editors of Tobacco Prevention \& Cessation would like to thank all our reviewers who have contributed to the journal in Volume 2 (2016).

AFFILIATION

1 Biomedical Research

Foundation of the Academy of Athens, Greece

2 European Network for Smoking and Tobacco

Prevention, Brussels, Belgium

CORRESPONDENCE TO

Prof. Panagiotis Behrakis. Chair of the Scientific Committee of European Network for Smoking and Tobacco Prevention, Brussels, Belgium. Email: panbehrakis@gmail.com
Redhwan Al-Naggar

Malaysia

MAli

United States

Jon-Patrick Allem

United States

\section{B Arnetz}

United States

\section{Umesh Aryal}

Nepal

\section{SR Bailey}

United States

George Bakhturidze

Georgia

Davut Baltaci

Turkey

\section{Ben Rejeb}

Tunisia

\section{Nazmi Bilir}

Turkey
Pierre Bizel

Belgium

Saif Borgan

Jordan

MC Brinkman

United States

Priscilla Callahan-Lyon

United States

Maria Cattaruzza

Italy

\section{Frank Chaloupka \\ United States}

\section{RD Chandora}

United States

\section{Marshall Cheney}

United States

\section{K Choi}

United States

\section{Luke Clancy}

Ireland

\section{Suzanne Colby}

United States

\section{Maria Cooper}

United States

\section{MR Creamer}

United States

\section{D'Ruiz}

United States

\section{Omar'Thanoon Dawood}

Malaysia

\section{NA Dietz}

United States

\section{JE Drehmer}

United States

\section{Erdöl \\ Turkey}

\section{KA Evans-Reeves}

United Kingdom 
M Fu

Spain

\section{Robert Garcia}

United States

A Gerber

Germany

Daniel Giovenco

United States

Charis Girvalaki

Greece

\section{Mary Gowin}

United States

\section{DA Groneberg}

Germany

WM Hajjar

Saudi Arabia

\section{Lisa Henriksen}

United States

\section{SY Ho}

Hong Kong

Carissa Baker Holmes

United States

\section{Nicole Hughes}

India

MJ Husain

United Kingdom

\section{Hamid Hussain}

Iraq

Jallow Isatou

United Kingdom

Radhakrishnan

Jayakrishnan

India
Dinesh Kaphle

South Africa

Paraskevi Katsaounou

Greece

B Kilibarda

Serbia

B King

Australia

\section{Grace Kong}

United States

NT Lam

Viet Nam

Anthony Laverty

United Kingdom

Joseph Lee

United States

YO Lee

United States

\section{Scott Leischow}

United States

S Levitz

Canada

David Levy

United States

Jeffrey Levy

United States

Brett Loomis

United States

\section{A Lopez}

Spain

Kate Mandeville

United Kingdom

\section{E Meijer}

Netherlands

Jawad Mohammed

United Kingdom

Paul Mowery

United States

F Naughton

United Kingdom

Pratibha Nayak

United States

Manfred Neuberger

Austria

Oluwakemi Odukoya

Nigeria

Adesola Olumide

Nigeria

Uyoyo Omaduvie

Nigeria

\section{Lanre Onigbogi}

Nigeria

\section{Sophia Papadakis}

Canada

T Patel

United States

Theodosia Peleki

Greece

Robert Philibert

United States

WB Pickworth

United States

Krzysztof Przewoniak

Poland 
Polosa Ricardo

Italy

\section{Nadeem Rizvi}

Pakistan

LM Romito

United States

\section{Hana Ross}

South Africa

\section{Rohini Ruhil}

India

\section{Maguy Saffouh El Hajj}

Qatar

\section{Jonathan Samet}

United States

\section{Roberta Savli}

Belgium

\section{SS Shaik}

India

\section{Gaurav Sharma}

India

\section{FC Sherratt}

United Kingdom

Danielle Smith

United States

\section{Chandrashekhar}

Sreeramareddy

Malaysia

\section{AW Stewart}

New Zealand

\section{P Stigler-Granados}

United States

\section{W Sumner}

United States
Prue Talbot

United States

Antigona Trofor

Romania

MC Tsai

Taiwan

Yasemin Turker

Turkey

\section{Jennifer Unger}

United States

\section{Sreenivas Vreeranki}

United States

\section{Theodore Wagener}

United States

\section{Kimberly Wagoner}

United States

\section{MPWang}

Hong Kong

\section{Thomas Wills}

United States

Pradeep K.Yadalam

India

\section{Nikola Zaharakis}

United States

H Zeeb

Germany 